Pacific

Journal of

Mathematics

\title{
BASES OF QUANTIZED ENVELOPING ALGEBRAS
}

\author{
BANGMING DENG AND JIE DU
}




\title{
BASES OF QUANTIZED ENVELOPING ALGEBRAS
}

\author{
BANGMING DENG AND JiE DU
}

\begin{abstract}
We give a systematic description of many monomial bases for a specified quantized enveloping algebra and of many integral monomial bases for the associated Lusztig $\mathbb{Z}\left[v, v^{-1}\right]$-form. The relations among monomial bases, PBW bases and canonical bases are also discussed.
\end{abstract}

\section{Introduction}

Let $\mathfrak{g}$ be a (complex) semisimple Lie algebra and let $\boldsymbol{U}^{+}$be the positive part of its associated quantized enveloping algebra $\boldsymbol{U}=\boldsymbol{U}_{v}(\mathfrak{g})$ over $\mathbb{Q}(v)$ with a DrinfeldJimbo presentation in the generators $E_{i}, F_{i}, K_{i}^{ \pm 1}(i \in I=[1, n])$. We denote by $U^{+}$the Lusztig form of $\boldsymbol{U}^{+}$, that is, $U^{+}$is generated by all the divided powers $E_{i}^{(m)}$ over $\mathscr{L}:=\mathbb{Z}\left[v, v^{-1}\right]$. Let $\Omega$ be the set of words on the alphabet $I$ and, for $w=i_{1}^{e_{1}} i_{2}^{e_{2}} \cdots i_{m}^{e_{m}} \in \Omega$ with $i_{j-1} \neq i_{j}$ for all $j$, put $E_{w}=E_{i_{1}}^{e_{1}} \cdots E_{i_{m}}^{e_{m}}$ and $\mathfrak{m}^{(w)}=E_{i_{1}}^{\left(e_{1}\right)} \cdots E_{i_{m}}^{\left(e_{m}\right)}$. Further, let $\Lambda$ denote the set of all functions from the set of positive roots of $\mathfrak{g}$ to nonnegative integers.

Certain monomial bases of the form $\mathfrak{m}^{(w)}$ have been introduced for $U^{+}$in [Lusztig 1990, 7.8] and [Ringel 1995, Theorem 1'] for the simply laced case, and in [Chari and Xi 1999] in general, and are used in the elementary construction of canonical bases. In this paper, we present a systematic way to sort out bases from the monomials $E_{w}$ for $\boldsymbol{U}^{+}$and from the monomials $\mathfrak{m}^{(w)}$ for $U^{+}$, and relate them to PBW bases and canonical bases. The main result is:

Theorem 1.1. Assume that $\mathfrak{g}$ is simply laced. There is a partition $\Omega=\bigcup_{\lambda \in \Lambda} \Omega_{\lambda}$ such that, by choosing an arbitrary word $w_{\lambda} \in \Omega_{\lambda}$ for every $\lambda \in \Lambda$, the set $\left\{E_{w_{\lambda}}\right\}_{\lambda \in \Lambda}$ of monomials forms a basis for $\boldsymbol{U}^{+}$. If all words $w_{\lambda}$ are chosen to be distinguished (see Section 5), the set $\left\{\mathfrak{m}^{\left(w_{\lambda}\right)}\right\}_{\lambda \in \Lambda}$ forms a $\mathscr{L}$-basis for $U^{+}$.

We shall see from Remarks 6.5 that the monomial bases given in [Lusztig 1990], [Ringel 1995] and [Reineke 2001a, 4.2] can be obtained in this systematic description by a selection of the representatives $w_{\lambda}$. The assumption of simply laced types

MSC2003: 17B37, 16G20.

Keywords: quantized enveloping algebra, Ringel-Hall algebra, generic extension, monomial basis, canonical basis.

Supported partially by the NSF of China (Grant no. 10271014), the TRAPOYP, the Doctoral Program of Higher Education, and the Australian Research Council. 
is made so that we may directly use the theory of quiver representations. See also [Deng and Du 2005] for a similar result in the affine $\mathfrak{s l}_{n}$ case. It is natural to expect that a similar result holds in the nonsimply laced case and to relate this theory to Kashiwara's crystal bases defined by using the monomials in Kashiwara operators [1991].

The main ingredients for the proof are Ringel's Hall algebra theory [Ringel 1995], the monoidal structure [Reineke 2001b] on the set $\mathcal{M}$ of isoclasses of finitedimensional representations of a Dynkin quiver $Q$ and the Bruhat-Chevalley type partial ordering on orbits in an affine space. These will be discussed separately in Sections 2, 3 and 4. Distinguished words are introduced and investigated in Section 5 and we prove the main result in Section 6. As an application of the theory, we mention an elementary construction [Reineke 2001b, §6] of the canonical bases for $U^{+}$as the counterpart of a similar construction for the Hecke algebra in [Kazhdan and Lusztig 1979]. This construction uses the same order as the one used in the geometric construction, involving perverse sheaf and intersection cohomology theories. Finally, more explicit results on distinguished words are worked out for the case of type $A$ in Section 7.

Throughout, $k$ denotes a finite field unless otherwise specified. Let $q_{k}=|k|$. All modules are finite-dimensional over $k$. If $M$ is a module, $n M, n \geqslant 0$, denotes the direct sum of n copies of $M$. Further, by $[M]$ we denote the class of modules isomorphic to $M$, i.e., the isoclass of $M$. For modules $M, N_{1}, \ldots, N_{t}$, let $F_{N_{1} \cdots N_{t}}^{M}$ denote the number of filtrations

$$
M=M_{0} \supset M_{1} \supset \cdots \supset M_{t-1} \supset M_{t}=0
$$

such that $M_{i-1} / M_{i} \cong N_{i}$ for all $1 \leqslant i \leqslant t$.

\section{Ringel-Hall algebras of Dynkin quivers}

Let $Q=\left(I, Q_{1}\right)$ be a quiver, i.e., a finite directed graph, where $I=Q_{0}$ is the set of vertices $\{1,2, \ldots, n\}$ and $Q_{1}$ is the set of arrows. If $\rho \in Q_{1}$ is an arrow from tail $i$ to head $j$, we write $h(\rho)$ for $j$ and $t(\rho)$ for $i$. Thus we obtain functions $h, t: Q_{1} \rightarrow I$. A vertex $i \in I$ is called a sink if there is no arrow $\rho$ with $t(\rho)=i$, and a source if there is no $\rho$ with $h(\rho)=i$.

Let $k Q$ be the path algebra of $Q$. A (finite-dimensional) representation $V$ of $Q$, consisting of a set of finite-dimensional vector spaces $V_{i}$ for each $i \in I$ and a set of linear transformations $V_{\rho}: V_{t(\rho)} \rightarrow V_{h(\rho)}$ for each $\rho \in Q_{1}$, is identified with a (left) $k Q$-module. We call $\operatorname{dim} V:=\left(\operatorname{dim} V_{1}, \ldots, \operatorname{dim} V_{n}\right)$ the dimension vector of $V$ and $\ell(V):=\sum_{i=1}^{n} \operatorname{dim} V_{i}$ the length of $V$ (also called the dimension of $V$ ). If 
$Q$ contains no oriented cycles, there are exactly $n$ pairwise nonisomorphic simple $k Q$-modules $S_{1}, \ldots, S_{n}$ corresponding bijectively to the vertices of $Q$.

From now on, we assume that $Q$ is a Dynkin quiver, that is, a quiver whose underlying graph is a (simply laced) Dynkin graph. By Gabriel's theorem [1972], there is a bijection between the set of isoclasses of indecomposable $k Q$-modules and a positive system $\Phi^{+}$of the root system $\Phi$ associated with $Q$. For any $\beta \in \Phi^{+}$, let $M(\beta)=M_{k}(\beta)$ denote the corresponding indecomposable $k Q$-module. By the Krull-Remak-Schmidt theorem, every $k Q$-module $M$ is isomorphic to

$$
M(\lambda)=M_{k}(\lambda):=\bigoplus_{\beta \in \Phi^{+}} \lambda(\beta) M_{k}(\beta),
$$

for some function $\lambda: \Phi^{+} \rightarrow \mathbb{N}$. Thus the isoclasses of $k Q$-modules are indexed by the set

$$
\Lambda=\left\{\lambda: \Phi^{+} \rightarrow \mathbb{N}\right\} \cong \mathbb{N}^{\mid \Phi^{+}} .
$$

By a result of Ringel [1990], for $\lambda, \mu_{1}, \ldots, \mu_{m}$ in $\Lambda$, there is a polynomial $\varphi_{\mu_{1} \cdots \mu_{m}}^{\lambda}(q) \in \mathbb{Z}[q]$, called a Hall polynomial, such that for any finite field $k$ of $q_{k}$ elements

$$
\varphi_{\mu_{1} \cdots \mu_{m}}^{\lambda}\left(q_{k}\right)=F_{M_{k}\left(\mu_{1}\right) \cdots M_{k}\left(\mu_{m}\right)}^{M_{k}(\lambda)} .
$$

Let $\mathscr{A}=\mathbb{Z}[q]$ be the integral polynomial ring in the indeterminate $q$. The generic (untwisted) Ringel-Hall algebra $\mathscr{H}=\mathscr{H}_{q}(Q)$ of $Q$ over $\mathscr{A}$ is by definition the free $\mathscr{A}$-module having basis $\left\{u_{\lambda} \mid \lambda \in \Lambda\right\}$, and satisfying the multiplicative relations

$$
u_{\mu} u_{\nu}=\sum_{\lambda \in \Lambda} \varphi_{\mu \nu}^{\lambda}(q) u_{\lambda}
$$

We sometimes write $u_{\lambda}=u_{[M(\lambda)]}$ in order to make certain calculations in term of modules. For $i \in I$, we set $u_{i}=u_{\left[S_{i}\right]}$. Clearly, $\mathscr{H}$ admits a natural $\mathbb{N}^{n}$-grading by dimension vectors.

Following [Ringel 1993b], we can twist the multiplication of the Ringel-Hall algebra to obtain the positive part $\boldsymbol{U}^{+}$of a quantized enveloping algebra.

Let $\mathscr{Z}=\mathbb{Z}\left[v, v^{-1}\right]$, where $v$ is an indeterminate with $v^{2}=q$. The twisted RingelHall algebra $\mathscr{H}^{\star}=\mathscr{H}_{v}^{\star}(Q)$ of $Q$ is by definition the free $\mathscr{Z}$-module having basis $\left\{u_{\lambda}=u_{[M(\lambda)]} \mid \lambda \in \Lambda\right\}$ and satisfying the multiplication rules

$$
u_{\mu} \star u_{v}=v^{\langle\mu, \nu\rangle} u_{\mu} u_{v}=v^{\langle\mu, v\rangle} \sum_{\lambda \in \Lambda} \varphi_{\mu \nu}^{\lambda}\left(v^{2}\right) u_{\lambda}
$$

where $\langle\mu, v\rangle=\operatorname{dim}_{k} \operatorname{Hom}_{k}(M(\mu), N(v))-\operatorname{dim}_{k} \operatorname{Ext}_{k Q}^{1}(M(\mu), N(v))$ is the Euler form associated with the quiver $Q$. Note that, if we define the bilinear form $\langle-,-\rangle$ : $\mathbb{Z}^{n} \times \mathbb{Z}^{n} \rightarrow \mathbb{Z}$ by

$$
\langle\boldsymbol{a}, \boldsymbol{b}\rangle=\sum_{i \in I} a_{i} b_{i}-\sum_{\rho \in Q_{1}} a_{t(\rho)} b_{h(\rho)},
$$


where $\boldsymbol{a}=\left(a_{1}, \cdots, a_{n}\right), \boldsymbol{b}=\left(b_{1}, \ldots, b_{n}\right)$, then

$$
\langle\mu, v\rangle=\langle\operatorname{dim} M(\mu), \operatorname{dim} M(v)\rangle .
$$

For each $m \geqslant 1$, set $[m]=\left(v^{m}-v^{-m}\right) /\left(v-v^{-1}\right)$ and $[m]^{!}=[1][2] \cdots[m]$. We define, for each $i \in I$, the divided powers

$$
u_{i}^{(\star m)}=\frac{u_{i}^{\star m}}{[m] !} \text { and } E_{i}^{(m)}=\frac{E_{i}^{m}}{[m] !}
$$

in $\mathscr{H}^{\star}$ and $\boldsymbol{U}^{+}$, respectively. Here $u_{i}^{\star m}=\underbrace{u_{i} \star \cdots \star u_{i}}_{m}=v^{m(m-1) / 2} u_{i}^{m}$.

Proposition 2.1 [Ringel 1995, §7]. The algebra $\mathscr{H}^{\star}$ is generated by all $u_{i}^{(\star m)}$, for $i \in I, m \geqslant 1$. There is a natural isomorphism

$$
\Psi: U^{+} \stackrel{\sim}{\rightarrow} \mathcal{H}^{\star}, E_{i}^{(m)} \mapsto u_{i}^{(\star m)} \quad(i \in I, m \geqslant 1) .
$$

We shall identify $U^{+}$with $\mathscr{H}^{\star}$ under this isomorphism.

\section{Generic extensions and the monoid $M$}

In this section, we collect some recent results on generic extensions for quiver representations over an algebraically closed field $k$.

Fix $\boldsymbol{d}=\left(d_{i}\right)_{i} \in \mathbb{N}^{n}$ and define the affine space

$$
R(\boldsymbol{d})=R(Q, \boldsymbol{d}):=\prod_{\alpha \in Q_{1}} \operatorname{Hom}_{k}\left(k^{d_{t(\alpha)}}, k^{d_{h(\alpha)}}\right) \cong \prod_{\alpha \in Q_{1}} k^{d_{h(\alpha)} \times d_{t(\alpha)}} .
$$

Thus a point $x=\left(x_{\alpha}\right)_{\alpha}$ of $R(\boldsymbol{d})$ determines a representation $V(x)$ of $Q$. The algebraic group $\mathrm{GL}(\boldsymbol{d})=\prod_{i=1}^{n} \mathrm{GL}_{d_{i}}(k)$ acts on $R(\boldsymbol{d})$ by conjugation:

$$
\left(g_{i}\right)_{i} \cdot\left(x_{\alpha}\right)_{\alpha}=\left(g_{h(\alpha)} x_{\alpha} g_{t(\alpha)}^{-1}\right)_{\alpha} .
$$

The GL(d)-orbits $O_{x}$ in $R(\boldsymbol{d})$ correspond bijectively to the isoclasses [V(x)] of representations of $Q$ with dimension vector $\boldsymbol{d}$.

The stabilizer GL $(\boldsymbol{d})_{x}=\{g \in \mathrm{GL}(\boldsymbol{d}) \mid g x=x\}$ of $x$ is the group of automorphisms of $M:=V(x)$ which is Zariski-open in $\operatorname{End}_{k}(M)$ and has dimension equal to $\operatorname{dim} \operatorname{End}_{k}(M)$. It follows that the orbit $O_{M}:=O_{x}$ of $M$ has dimension

$$
\operatorname{dim} O_{M}=\operatorname{dim} \operatorname{GL}(\boldsymbol{d})-\operatorname{dim} \operatorname{End}_{k}(M) .
$$

Lemma 3.1 [Reineke 2001b]. Let $Q$ be a Dynkin quiver. For $x \in R\left(\boldsymbol{d}_{1}\right)$ and $y \in R\left(\boldsymbol{d}_{2}\right)$, let $\mathscr{E}\left(\mathrm{O}_{x}, O_{y}\right)$ be the set of all $z \in R(\boldsymbol{d})$ where $\boldsymbol{d}=\boldsymbol{d}_{1}+\boldsymbol{d}_{2}$ such that $V(z)$ is an extension of some $M \in \mathcal{O}_{x}$ by some $N \in \mathbb{O}_{y}$. Then $\mathscr{E}\left(\mathbb{O}_{x}, O_{y}\right)$ is irreducible. 
Given representations $M, N$ of $Q$, consider the extensions

$$
0 \rightarrow N \rightarrow E \rightarrow M \rightarrow 0
$$

of $M$ by $N$. By the lemma, there is a unique (up to isomorphism) such extension $G$ with $\operatorname{dim} O_{G}$ maximal (i.e., with $\operatorname{dim} \operatorname{End}_{k}(G)$ minimal). We call $G$ the generic extension of $M$ by $N$, denoted by $M * N$.

For two representations $M, N$, we say that $M$ degenerates to $N$, or that $N$ is a degeneration of $M$, and write $[N] \leqslant[M]$ (or simply $N \leqslant M$ ), if $\mathscr{O}_{N} \subseteq \overline{\mathrm{O}}_{M}$, the closure of $\mathscr{O}_{M}$. Note that $N<M \Longleftrightarrow \mathcal{O}_{N} \subseteq \overline{\mathrm{O}}_{M} \backslash \mathrm{O}_{M}$.

Remark 3.2. The relation $\leqslant$ on the isoclasses is independent of the field $k$. This is seen from the following equivalence proved in [Bongartz 1996, Proposition 3.2]:

$$
N \leqslant M \Longleftrightarrow \operatorname{dim} \operatorname{Hom}(X, N) \geqslant \operatorname{dim} \operatorname{Hom}(X, M) \text { for all } X
$$

and the fact that the dimension $\operatorname{dim} \operatorname{Hom}(X, Y)$ is the same over any field. Thus we may simply define a (characteristic-free) partial order on $\Lambda$ by

$$
\lambda \leqslant \mu \Longleftrightarrow M_{k}(\lambda) \leqslant M_{k}(\mu) .
$$

for any given (algebraically closed) field $k$.

The first part of the following result is well-known (see, for example, [Bongartz 1996, 1.1]) and the other parts are proved in [Reineke 2001b].

Theorem 3.3. (1) If $0 \rightarrow N \rightarrow E \rightarrow M \rightarrow 0$ is exact and nonsplit, then $M \oplus N<E$.

(2) Let $M, N, X$ be representations of $Q$. Then $X \leqslant M * N$ if and only if there exist $M^{\prime} \leqslant M, N^{\prime} \leqslant N$ such that $X$ is an extension of $M^{\prime}$ by $N^{\prime}$. In particular, $M^{\prime} \leqslant M, N^{\prime} \leqslant N \Longrightarrow M^{\prime} * N^{\prime} \leqslant M * N$.

(3) Let $M$ be the set of isoclasses of $k Q$-modules and define a multiplication * on $\mathcal{M}$ by $[M] *[N]=[M * N]$ for any $[M],[N] \in \mathcal{M}$. Then $\mathcal{M}$ is a monoid with identity $1=[0]$ and the multiplication $*$ preserves the induced partial ordering on $M$.

(4) $M$ is generated by the simple modules $\left[S_{i}\right], i \in I$.

Let $\Omega$ be the set of words in the alphabet $I=\{1, \ldots, n\}$. For $w=i_{1} i_{2} \cdots i_{m} \in \Omega$, let $\wp(w) \in \Lambda$ be the element defined by

$$
\left[S_{i_{1}}\right] * \cdots *\left[S_{i_{m}}\right]=[M(\wp(w))] .
$$

Thus we obtain a map $\wp: \Omega \rightarrow \Lambda$. The theorem shows that $\wp$ is surjective and induces a partition $\Omega=\bigcup_{\lambda \in \Lambda} \Omega_{\lambda}$ with $\Omega_{\lambda}=\wp^{-1}(\lambda)$. Each $\Omega_{\lambda}$ is called a fibre of $\wp$.

By Remark 3.2, if we set $\lambda * \mu:=M(\lambda * \mu) \cong M(\lambda) * M(\mu)$ for $\lambda, \mu \in \Lambda$, the element $\lambda * \mu$ is well-defined, independent of the field $k$. Note that the multiplication $*$ on $\Lambda$ depends on the orientation of $Q$. 


\section{The poset $\Lambda$}

In this section we look at some properties of the poset $(\Lambda, \leqslant)$, where $\leqslant$ is defined in Remark 3.2.

For $w=i_{1} i_{2} \cdots i_{m} \in \Omega$ and $\lambda \in \Lambda$, let $\varphi_{w}^{\lambda}$ denote the Hall polynomial $\varphi_{\mu_{1} \cdots \mu_{m}}^{\lambda}$, where $M\left(\mu_{r}\right) \cong S_{i_{r}}$. Thus, for a finite field $k$,

$$
\varphi_{w}^{\lambda}\left(q_{k}\right)=F_{S_{i_{1}} \cdots S_{i_{m} k}}^{M_{k}(\lambda)}
$$

is the number of composition series of $M_{k}(\lambda)$ :

$$
M_{k}(\lambda)=M_{0} \supset M_{1} \supset \cdots \supset M_{m-1} \supset M_{m}=0
$$

with $M_{j-1} / M_{j} \cong S_{i_{j} k}$. Such a composition series is called a composition series of type $w$.

The following lemma is a bit stronger than [Deng and Du 2005, 6.2].

Lemma 4.1. Let $w \in \Omega$ and $\mu \geqslant \lambda$ in $\Lambda$. Then $\varphi_{w}^{\mu} \neq 0$ implies $\varphi_{w}^{\lambda} \neq 0$.

Proof. Let $w=i_{1} i_{2} \cdots i_{m}$ and $w^{\prime}=i_{2} \cdots i_{m}$. We apply induction on $m$. If $m=1$ then $\mu \geqslant \lambda$ forces $M(\mu)=M(\lambda)$ and the result is clear. Now assume $m>1$. If $\varphi_{w}^{\mu} \neq 0$, then $\varphi_{w}^{\mu}\left(q_{k}\right) \neq 0$ for some finite field $k$. Thus $M_{k}(\mu)$ has a submodule $M_{k}^{\prime} \cong M_{k}\left(\mu^{\prime}\right)$ having a composition series of type $w^{\prime}$. Hence $\varphi_{w^{\prime}}^{\mu^{\prime}} \neq 0$, since $\varphi_{w^{\prime}}^{\mu^{\prime}}\left(q_{k}\right) \neq 0$. Base change to the algebraic closure $\bar{k}$ of $k$ gives an exact sequence over $\bar{k}$

$$
0 \longrightarrow M^{\prime} \longrightarrow M(\mu) \longrightarrow S_{i_{1}} \longrightarrow 0,
$$

where we have dropped the subscripts $\bar{k}$. Thus

$$
M(\lambda) \leqslant M(\mu) \leqslant S_{i_{1}} * M^{\prime} .
$$

By Theorem 3.3(2), there exist modules $N^{\prime}, N^{\prime \prime}$ such that $M(\lambda)$ is an extension of $N^{\prime}$ by $N^{\prime \prime}$ and $N^{\prime} \leqslant M^{\prime}, N^{\prime \prime} \leqslant S_{i_{1}}$. So we obtain an exact sequence (over $\bar{k}$ )

$$
0 \longrightarrow N^{\prime} \stackrel{f}{\longrightarrow} M(\lambda) \stackrel{g}{\longrightarrow} N^{\prime \prime} \longrightarrow 0 .
$$

Now the condition $N^{\prime} \leqslant M^{\prime}$ means $\lambda^{\prime} \leqslant \mu^{\prime}$ where $N^{\prime} \cong M\left(\lambda^{\prime}\right)$. Since $\varphi_{w^{\prime}}^{\mu^{\prime}} \neq 0$, it follows from induction that $\varphi_{w^{\prime}}^{\lambda^{\prime}} \neq 0$, that is, $N^{\prime}$ has a composition series of type $w^{\prime}$. On the other hand, since $S_{i_{1}}$ is simple, $N^{\prime \prime} \leqslant S_{i_{1}}$ implies $N^{\prime \prime} \cong S_{i_{1}}$. Therefore, $M(\lambda)$ has a composition series of type $w$, and consequently, $\varphi_{w}^{\lambda} \neq 0$.

We now relate the partial order $\leqslant$ to certain nonzero Hall polynomials.

Theorem 4.2. Let $\lambda, \mu \in \Lambda$. Then $\lambda \leqslant \mu$ if and only if there exists a word $w \in$ $\wp^{-1}(\mu)$ with $\varphi_{w}^{\lambda} \neq 0$. 
Proof. Suppose $\lambda \leqslant \mu$. Since $\wp$ is surjective, $\mu=\wp(w)$ for some $w \in \Omega$. By (3-2), we see that $\varphi_{w}^{\wp(w)} \neq 0$. Thus Lemma 4.1 implies $\varphi_{w}^{\lambda} \neq 0$, as required.

Conversely, let $w=i_{1} i_{2} \cdots i_{m} \in \Omega, \lambda \in \Lambda$, and suppose $\varphi_{w}^{\lambda} \neq 0$. We use induction on $m$ to prove that $\lambda \leqslant \wp(w)$. If $m=1$, there is nothing to prove. Let $m>1$ and $w^{\prime}=i_{2} \cdots i_{m}$ and assume $\lambda^{\prime} \leqslant \wp\left(w^{\prime}\right)$ whenever $\varphi_{w^{\prime}}^{\lambda^{\prime}} \neq 0$. Since $\varphi_{w}^{\lambda} \neq 0$, there is a finite field $k$ (of any given characteristic) such that $\varphi_{w}^{\lambda}\left(q_{k}\right) \neq 0$. Thus there is a submodule $M_{k}^{\prime}$ of $M_{k}(\lambda)$ having a composition series of type $w^{\prime}$. This implies $\varphi_{w^{\prime}}^{\lambda^{\prime}} \neq 0$ where $M_{k}\left(\lambda^{\prime}\right) \cong M_{k}^{\prime}$. By induction, we have $\lambda^{\prime} \leqslant \wp\left(w^{\prime}\right)$.

On the other hand, base change to the exact sequence

$$
0 \longrightarrow M_{k}^{\prime} \longrightarrow M_{k}(\lambda) \longrightarrow S_{i_{1} k} \longrightarrow 0
$$

yields an exact sequence over $\bar{k}$

$$
0 \longrightarrow M^{\prime} \longrightarrow M(\lambda) \longrightarrow S_{i_{1}} \longrightarrow 0 \text {. }
$$

(Here again we dropped the subscripts $\bar{k}$.) By Theorem 3.3(2) we obtain

$$
M(\lambda) \leqslant S_{i_{1}} * M\left(\lambda^{\prime}\right) \leqslant S_{i_{1}} * M\left(\wp\left(w^{\prime}\right)\right)=M(\wp(w)) .
$$

Therefore, $\lambda \leqslant \wp(w)$.

\section{Distinguished words}

Let $w=i_{1} i_{2} \cdots i_{m}$ be a word in $\Omega$. Then $w$ can be uniquely expressed in the tight form $w=j_{1}^{e_{1}} j_{2}^{e_{2}} \cdots j_{t}^{e_{t}}$, where $e_{r} \geqslant 1,1 \leqslant r \leqslant t$, and $j_{r} \neq j_{r+1}$ for $1 \leqslant r \leqslant t-1$. Following [Ringel 1993a, 2.3], a filtration

$$
M=M_{0} \supset M_{1} \supset \cdots \supset M_{t-1} \supset M_{t}=0
$$

of a module is called a reduced filtration of type $w$ if $M_{r-1} / M_{r} \cong e_{r} S_{j_{r}}$ for all $1 \leqslant r \leqslant t$. Any reduced filtration of $M$ of type $w$ can be refined to a composition series of $M$ of type $w$. Conversely, given a composition series of $M$ of type $w$, there is a unique reduced filtration of $M$ of type $w$ such that the given composition series is a refinement of this reduced filtration. By $\gamma_{w}^{\lambda}(q)$ we denote the Hall polynomial $\varphi_{\mu_{1} \cdots \mu_{t}}^{\lambda}(q)$, where $M\left(\mu_{r}\right)=e_{r} S_{j_{r}}$. Thus, for a finite field $k$ of $q_{k}$ elements, $\gamma_{w}^{\lambda}\left(q_{k}\right)$ is the number of the reduced filtrations of $M_{k}(\lambda)$ of type $w$. A word $w$ is called distinguished if $\gamma_{w}^{\wp(w)}(q)=1$; this is the case if and only if, for some algebraically closed field $k, M_{k}(\wp(w))$ has a unique reduced filtration of type $w$. See [Deng and Du 2005, §5].

Example 5.1. Let $w=j_{1}{ }^{e_{1}} j_{2}{ }^{e_{2}} \cdots j_{t}{ }^{e_{t}}$ be in the tight form. If $j_{1}, \ldots, j_{t}$ are pairwise distinct and satisfy

$$
\operatorname{Ext}_{k Q}^{1}\left(S_{j_{r}}, S_{j_{s}}\right) \neq 0 \Longrightarrow r<s
$$


then $F_{N_{1} \cdots N_{t}}^{M}=0$ or 1 for every $k Q$-module $M$, where $N_{r}=e_{r} S_{j_{r}}$. Thus $w$ is distinguished.

Distinguished words will be used in the construction of integral monomial bases for the Lusztig form. The following lemma shows that these words are somehow evenly distributed.

Lemma 5.2. Each fibre of $\wp$ contains at least one distinguished word.

Proof. This follows directly from [Reineke 2001a, Lemma 4.5]. For completeness, we present here the construction of such distinguished words.

By $\mathscr{I}$ we denote the set of the isoclasses of indecomposable representations of $Q$. Let $\mathscr{I}_{*}$ be a directed partition of $\mathscr{I}$ [Reineke 2001a, §4], that is, a partition of the set $\mathscr{I}$ into subsets $\mathscr{I}_{1}, \ldots, \mathscr{I}_{m}$ such that

(a) $\operatorname{Ext}_{k Q}^{1}(M, N)=0$ for all $M, N$ in the same part $\Phi_{r}$,

(b) $\operatorname{Ext}_{k Q}^{1}(M, N)=0=\operatorname{Hom}_{k} Q(N, M)$ if $M \in \mathscr{I}_{r}, N \in \mathscr{I}_{s}$, where $1 \leqslant r<s \leqslant m$.

Then, for each $\lambda \in \Lambda$, we have a unique decomposition

$$
M(\lambda)=\bigoplus_{r=1}^{m} M_{r},
$$

where all the summands of $M_{r}$ belong to $\Phi_{r}, 1 \leqslant r \leqslant m$. Thus

$$
\operatorname{Hom}_{k}\left(M_{r}, M_{s}\right) \neq 0 \Longrightarrow r \leqslant s .
$$

Further, since $Q$ is a Dynkin quiver, we can order the vertices of $Q$ in a sequence $i_{1}, i_{2}, \ldots, i_{n}$ such that, for each $1<j \leqslant n, i_{j}$ is a sink in the full subquiver of $Q$ with vertices $\left\{i_{1}, \ldots, i_{j-1}, i_{j}\right\}$. Equivalently, $i_{1}, i_{2}, \ldots, i_{n}$ are ordered to satisfy

$$
\operatorname{Ext}_{k Q}^{1}\left(S_{i_{j}}, S_{i_{l}}\right) \neq 0 \Longrightarrow j<l .
$$

Let $\boldsymbol{d}^{(r)}=\left(d_{1}^{(r)}, \ldots, d_{n}^{(r)}\right)=\operatorname{dim} M_{r}$, for $1 \leqslant r \leqslant m$, and set

$$
w_{r}=\underbrace{i_{1} \cdots i_{1}}_{d_{i_{1}}^{(r)}} \cdots \cdots \underbrace{i_{n} \cdots i_{n}}_{d_{i_{n}}^{(r)}}
$$

and $w_{\lambda}=w_{1} \cdots w_{m} \in \Omega$. Then [Reineke 2001a, Lemma 4.5] implies that $\wp\left(w_{\lambda}\right)=$ $\lambda$ and $\gamma_{w_{\lambda}}^{\lambda}(q)=1$, that is, $w_{\lambda}$ is distinguished.

We call the distinguished words constructed above directed distinguished words (with respect to the given directed partition $\mathscr{I}_{*}$ ).

We mention a special case of directed partitions $\Phi_{*}$ where each part $\mathscr{I}_{r}$ contains only one isoclass. This case is equivalent to ordering the indecomposable modules $M\left(\beta_{1}\right), M\left(\beta_{2}\right), \ldots$ such that

$$
\operatorname{Hom}_{k}\left(M\left(\beta_{r}\right), M\left(\beta_{s}\right)\right) \neq 0 \Longrightarrow r \leqslant s .
$$


Note that monomial bases associated to these special directed distinguished words have been constructed in [Lusztig 1990] and [Ringel 1995]; see Remarks 6.5 below.

The following example shows that a fibre of $\wp$ could contain many words other than directed distinguished ones.

Example 5.3. Let $Q$ denote the quiver

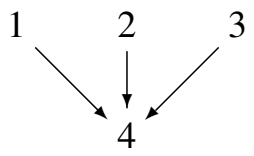

Let $\lambda \in \Lambda$ be such that $M(\lambda)$ is the indecomposable $k Q$-module with dimension vector $(1,1,1,2)$. Then $\wp^{-1}(\lambda)$ contains 12 words

$$
\begin{aligned}
& 1234^{2}, 1324^{2}, 2134^{2}, 2314^{2}, 3124^{2}, 3214^{2}, \\
& 12434,13424,21434,23414,31424,32414
\end{aligned}
$$

all distinguished. From the structure of the Auslander-Reiten quiver of $k Q$, one sees easily that the first 6 words are directed distinguished, but the last 6 are not.

\section{Monomial and integral monomial bases}

For $m \geqslant 1$, let $\llbracket m \rrbracket^{!}=\llbracket 1 \rrbracket \llbracket 2 \rrbracket \cdots \llbracket m \rrbracket$, where $\llbracket e \rrbracket=\left(q^{e}-1\right) /(q-1)$. Then $\llbracket m \rrbracket=$ $v^{m-1}[m]$ and $\llbracket m \rrbracket^{!}=v^{m(m-1) / 2}[m]^{!}$.

Lemma 6.1. Let $w \in \Omega$ be a word with the tight form $j_{1}^{e_{1}} j_{2}^{e_{2}} \ldots j_{t}^{e_{t}}$. Then, for each $\lambda \in \Lambda$,

$$
\varphi_{w}^{\lambda}(q)=\gamma_{w}^{\lambda}(q) \prod_{r=1}^{t} \llbracket e_{r} \rrbracket^{!} .
$$

In particular, $\varphi_{w}^{\wp(w)}(q)=\prod_{r=1}^{t} \llbracket e_{r} \rrbracket^{!}$if $w$ is distinguished.

Proof. The result follows from the definition of a distinguished word and the fact that the number of composition series of $e S_{i}$ is $\llbracket e \rrbracket !$ (see [Ringel 1993b, 8.2]).

To each word $w=i_{1} i_{2} \cdots i_{m} \in \Omega$, we associate a monomial

$$
u_{w}=u_{i_{1}} u_{i_{2}} \cdots u_{i_{m}} \in \mathcal{H} .
$$

Theorem 4.2 and Lemma 6.1 give:

Proposition 6.2. For each $w \in \Omega$ with the tight form $j_{1}{ }^{e_{1}} j_{2}{ }^{e_{2}} \cdots j_{t}{ }^{e_{t}}$, we have

$$
u_{w}=\sum_{\lambda \leqslant \wp(w)} \varphi_{w}^{\lambda}(q) u_{\lambda}=\prod_{r=1}^{t} \llbracket e_{r} \rrbracket^{!} \sum_{\lambda \leqslant \wp(w)} \gamma_{w}^{\lambda}(q) u_{\lambda} .
$$

Moreover, the coefficients appearing in the sum are all nonzero. 
This improves [Ringel 1995, Theorem 1, p. 96] in two ways: it generalizes the formula from certain directed distinguished words to all words, and it replaces the lexicographical order by the Bruhat type partial order $\leqslant$.

For any commutative ring $\mathscr{A}^{\prime}$ which is an $\mathscr{A}$-algebra and any $\mathscr{A}$-module $M$, let $M_{\mathscr{A}}=\mathscr{A}^{\prime} \otimes_{\mathscr{A}} M$ denote the $\mathscr{A}^{\prime}$-module obtained from $M$ by base change to $\mathscr{A}^{\prime}$.

Theorem 6.3. For every $\lambda \in \Lambda$, choose an arbitrary word $w_{\lambda} \in \wp^{-1}(\lambda)$. The set $\left\{u_{w_{\lambda}} \mid \lambda \in \Lambda\right\}$ is a $\mathbb{Q}(q)$-basis of $\mathscr{H}_{\mathbb{Q}(q)}$. If all the $w_{\lambda}$ are chosen to be distinguished, then this set is an $\mathscr{A}_{(q-1)}$-basis of $\mathscr{H}_{\mathscr{A}_{(q-1)}}$ where $\mathscr{A}_{(q-1)}$ denotes the localization of $A$ at the maximal ideal generated by $q-1$.

Proof. This follows from Proposition 6.2 and the fact that $\varphi_{w_{\lambda}}^{\wp\left(w_{\lambda}\right)}$ is invertible in $\mathscr{A}_{(q-1)}$ if $w_{\lambda}$ is distinguished.

Let $\mathfrak{g}=\mathfrak{n}_{-} \oplus \mathfrak{h} \oplus \mathfrak{n}_{+}$be the Lie algebra over $\mathbb{Q}$ of type $Q$ with generators $e_{i}, f_{i}, h_{i}$. Let $\mathfrak{U}(\mathfrak{g})$ be the universal enveloping algebra of $\mathfrak{g}$. Define monomials $e_{w}$ similarly for $w \in \Omega$ in $\mathfrak{U}\left(\mathfrak{n}_{+}\right)$.

Corollary 6.4. For every $\lambda \in \Lambda$, choose an arbitrary distinguished word $w_{\lambda} \in$ $\wp^{-1}(\lambda)$. The set $\left\{e_{w_{\lambda}} \mid \lambda \in \Lambda\right\}$ is a $\mathbb{Q}$-basis of $\mathfrak{U}\left(\mathfrak{n}_{+}\right)$.

Proof. The result follows from the isomorphism $\mathscr{H}_{\mathscr{A}^{\prime}} /(q-1) \mathscr{H}_{\mathscr{A}^{\prime}} \cong \mathfrak{U}\left(\mathfrak{n}_{+}\right)$, where $\mathscr{A}^{\prime}=\mathscr{A}_{(q-1)}$, and Theorem 6.3.

Proof of Theorem 1.1. For each $w=i_{1} i_{2} \cdots i_{m} \in \Omega$ we have

$$
u_{i_{1}} \star \cdots \star u_{i_{m}}=v^{\varepsilon(w)} u_{w},
$$

where

$$
\varepsilon(w)=\sum_{1 \leqslant r<s \leqslant m}\left\langle\operatorname{dim} S_{i_{r}}, \operatorname{dim} S_{i_{s}}\right\rangle .
$$

Let, for $w=j_{1}^{e_{1}} \cdots j_{t}^{e_{t}}$ in tight form,

$$
\mathfrak{m}^{(w)}:=E_{j_{1}}^{\left(e_{1}\right)} \cdots E_{j_{t}}^{\left(e_{t}\right)}=\left(\prod_{r=1}^{t}\left[e_{r}\right]^{!}\right)^{-1} u_{j_{1}}^{\star e_{1}} \star \cdots \star u_{j_{t}}^{\star e_{t}} .
$$

Since $\prod_{r=1}^{t}\left[e_{r}\right]^{!}=v^{-\delta(w)} \prod_{r=1}^{t} \llbracket e_{r} \rrbracket^{!}$, where $\delta(w)=\sum_{r=1}^{t} e_{r}\left(e_{r}-1\right) / 2$, it follows from Proposition 6.2 that

$$
\mathfrak{m}^{(w)}=\left(\prod_{r=1}^{t} \llbracket e_{r} \rrbracket^{!}\right)^{-1} v^{\delta(w)+\varepsilon(w)} u_{w}=v^{\delta(w)+\varepsilon(w)} \sum_{\lambda \leqslant \wp(w)} \gamma_{w}^{\lambda}\left(v^{2}\right) u_{\lambda} .
$$

Together with Proposition 2.1 and Theorem 6.3, this implies Theorem 1.1 with $\Omega_{\lambda}=\wp^{-1}(\lambda)$ for all $\lambda \in \Lambda$. 
Remarks 6.5. (a) It is clear, from the definition, that the monomial basis $\left\{E^{(M)}\right\}$ constructed in [Reineke 2001a, Theorem 4.2] involves only directed distinguished words $w_{\lambda}$.

(b) As a special case of [Reineke 2001a, Theorem 4.2], the monomial bases constructed in [Lusztig 1990, 7.8; Ringel 1995, pp. 101-2] involve only those directed distinguished words defined with respect to the special directed partition $\mathscr{I}_{*}$ satisfying conditions (5-3) and (5-2); see [Ringel 1995, Theorem 1] and [Lusztig 1990, 4.12(c), 4.13]. ${ }^{1}$

We now look briefly at the elementary and algebraic construction of the canonical basis for $U^{+}$[Reineke 2001b, §6]. Note that the elementary constructions given in, e.g., [Lusztig 1990; Kashiwara 1991; Ringel 1995; Chari and Xi 1999] used a finer order than the one used in the geometric construction. We now use the same order which has an algebraic interpretation (3-1).

For each $\lambda \in \Lambda$, set

$$
\tilde{\mathfrak{u}}_{\lambda}=v^{-\operatorname{dim} M(\lambda)+\operatorname{dim} \operatorname{End}(M(\lambda))} u_{\lambda} .
$$

Then, by Proposition $2.1, U^{+}$is $\mathscr{Z}$-free with basis $\mathscr{E}=\left\{\tilde{\mathfrak{u}}_{\lambda}: \lambda \in \Lambda\right\}$. Note that $U^{+}=\bigoplus_{\boldsymbol{d}} U_{\boldsymbol{d}}^{+}$is $\mathbb{N} I$-graded according to the dimension vectors, and each $U_{\boldsymbol{d}}^{+}$is $\mathscr{L}$-free with basis $\mathscr{E} \cap U_{\boldsymbol{d}}^{+}=\left\{\tilde{\mathfrak{u}}_{\lambda}: \lambda \in \Lambda_{\boldsymbol{d}}\right\}$. Clearly, each $\Lambda_{\boldsymbol{d}}$ together with $\leqslant$ is a poset.

Define a ring homomorphism $\iota: U^{+} \rightarrow U^{+}$by setting $\iota\left(E_{i}^{(m)}\right)=E_{i}^{(m)}$ and $\iota(v)=v^{-1}$. Clearly, $\iota$ preserves the grading of $U^{+}$. Write, for any $\tilde{\mathfrak{u}}_{\lambda} \in U_{\boldsymbol{d}}^{+}$,

$$
\iota\left(\tilde{\mathfrak{u}}_{\mu}\right)=\sum_{\lambda} r_{\lambda, \mu} \tilde{\mathfrak{u}}_{\lambda}
$$

By [Lusztig 1990, 9.10] (see [Du 1994] for more details), the existence of the canonical bases for $U_{\boldsymbol{d}}^{+}$follows from the property

$$
r_{\lambda, \lambda}=1, r_{\lambda, \mu}=0 \quad \text { unless } \lambda \leqslant \mu .
$$

of the coefficients $r_{\lambda, \mu}$. We use (6-2) to derive (6-4). We first calculate $\delta(w)+\varepsilon(w)$ for directed distinguished words; compare [Ringel 1995, Lemma, p. 102].

Lemma 6.6. We have for any directed distinguished word $w \in \Omega$

$$
\delta(w)+\varepsilon(w)=-\operatorname{dim} M(\wp(w))+\operatorname{dim} \operatorname{End} M(\wp(w)) .
$$

Proof. Let $w \in \Omega$ be a directed distinguished word. Then, by definition, there is a directed partition $\mathscr{I}_{*}$ of $\mathscr{I}$ and a $\lambda \in \Lambda$ such that $w$ has the form $w=w_{\lambda}=w_{1} \cdots w_{m}$

\footnotetext{
${ }^{1}$ It seems to us that condition (5-2) was implicitly used in [Lusztig 1990, 7.2], though it was not explicitly stated in the paper.
} 
with

$$
w_{r}=\underbrace{i_{1} \cdots i_{1}}_{d_{i_{1}}^{(r)}} \cdots \cdots \underbrace{i_{n} \cdots i_{n}}_{d_{i_{n}}^{(r)}},
$$

where $M(\lambda)=M_{1} \oplus M_{2} \oplus \cdots \oplus M_{m}, \boldsymbol{d}^{(r)}=\left(d_{1}^{(r)}, \ldots, d_{n}^{(r)}\right)=\operatorname{dim} M_{r}$ for $1 \leqslant r \leqslant m$, and the sequence $i_{1}, i_{2}, \ldots, i_{n}$ of vertices are ordered to satisfy (5-2). Clearly,

$$
\delta(w)=\sum_{r=1}^{m} \sum_{j=1}^{n} \frac{d_{i_{j}}^{(r)}\left(d_{i_{j}}^{(r)}-1\right)}{2} .
$$

Since $\left\langle\operatorname{dim} S_{i_{j}}, \operatorname{dim} S_{i_{l}}\right\rangle=0$ for $j>l$ and $\operatorname{Ext}^{1}\left(M_{r}, M_{s}\right)=0$ for all $1 \leqslant r \leqslant s \leqslant m$, we obtain, for each $1 \leqslant r \leqslant m$,

$$
\begin{aligned}
\varepsilon\left(w_{r}\right) & =\sum_{j=1}^{n} \frac{d_{i_{j}}^{(r)}\left(d_{i_{j}}^{(r)}-1\right)}{2}\left\langle\operatorname{dim} S_{i_{j}}, \operatorname{dim} S_{i_{j}}\right\rangle+\sum_{1 \leqslant j<l \leqslant n}\left\langle\operatorname{dim} d_{i_{j}}^{(r)} S_{i_{j}}, \operatorname{dim} d_{i_{l}}^{(r)} S_{i_{l}}\right\rangle \\
& =\left\langle\operatorname{dim} M_{r}, \operatorname{dim} M_{r}\right\rangle-\sum_{j=1}^{n} \frac{\left(d_{i_{j}}^{(r)}\right)^{2}}{2}-\sum_{j=1}^{n} \frac{d_{i_{j}}^{(r)}}{2} \\
& =\operatorname{dim} \operatorname{End}\left(M_{r}\right)-\sum_{j=1}^{n} \frac{d_{i_{j}}^{(r)}\left(d_{i_{j}}^{(r)}+1\right)}{2}
\end{aligned}
$$

and therefore,

$$
\begin{aligned}
\varepsilon(w) & =\sum_{r=1}^{m} \varepsilon\left(w_{r}\right)+\sum_{1 \leqslant r<s \leqslant m}\left\langle\operatorname{dim} M_{r}, \operatorname{dim} M_{s}\right\rangle \\
& =\sum_{r=1}^{m} \varepsilon\left(w_{r}\right)+\sum_{1 \leqslant r<s \leqslant m} \operatorname{dim} \operatorname{Hom}\left(M_{r}, M_{s}\right) .
\end{aligned}
$$

Noting that $\operatorname{Hom}\left(M_{r}, M_{s}\right)=0$ for $r>s$, we finally obtain

$$
\begin{aligned}
\delta(w)+\varepsilon(w) & =\sum_{r=1}^{m} \operatorname{dim} \operatorname{End}\left(M_{r}\right)+\sum_{1 \leqslant r<s \leqslant m} \operatorname{dim} \operatorname{Hom}\left(M_{r}, M_{s}\right)-\sum_{r=1}^{m} \sum_{j=1}^{n} d_{i_{j}}^{(r)} \\
& =\operatorname{dim} \operatorname{End}(M(\lambda))-\operatorname{dim} M(\lambda) .
\end{aligned}
$$

This completes the proof.

By Lemma 6.6 and (6-2), any directed distinguished word $w$ satisfies

$$
\mathfrak{m}^{(w)}=\tilde{\mathfrak{u}}_{\wp(w)}+\sum_{\lambda<\wp(w)} f_{\lambda, \wp(w)} \tilde{\mathfrak{u}}_{\lambda}
$$


where $0 \neq f_{\lambda, \wp(w)} \in \mathscr{L}$. If we fix a representative set $\Lambda^{\prime}=\left\{w_{\lambda}: \lambda \in \Lambda\right\}$, where $w_{\lambda} \in \Omega_{\lambda}$, consisting of directed distinguished words, the relation above implies that, for any $\mu \in \Lambda$,

$$
\tilde{\mathfrak{u}}_{\mu} \in \mathfrak{m}^{\left(w_{\mu}\right)}+\sum_{\lambda<\mu} \mathscr{L}^{\left(w_{\lambda}\right)} .
$$

Restricting to $\Lambda_{\boldsymbol{d}}$, where $\boldsymbol{d}$ is a fixed dimension vector, we obtain the transition matrix $\left(f_{\lambda, \mu}\right)_{\lambda, \mu \in \Lambda_{d}}$. This matrix has an inverse $\left(g_{\lambda, \mu}\right)_{\lambda, \mu \in \Lambda_{d}}$ satisfying $g_{\lambda, \lambda}=1$ and $g_{\lambda, \mu}=0$ unless $\lambda \leqslant \mu$. Thus

$$
\tilde{\mathfrak{u}}_{\mu}=\mathfrak{m}^{\left(w_{\mu}\right)}+\sum_{\lambda<\mu} g_{\lambda, \mu} \mathfrak{m}^{\left(w_{\lambda}\right)}
$$

Applying $\iota$, we obtain by (6-5)

$$
\iota\left(\tilde{\mathfrak{u}}_{\mu}\right)=\mathfrak{m}^{\left(w_{\mu}\right)}+\sum_{\lambda<\mu} \bar{g}_{\lambda, \mu} \mathfrak{m}^{\left(w_{\lambda}\right)}=\tilde{\mathfrak{u}}_{\mu}+\sum_{\lambda<\mu} r_{\lambda, \mu} \tilde{\mathfrak{u}}_{\lambda} .
$$

This proves that the coefficients in (6-3) satisfy (6-4). Thus the corresponding canonical basis $\left\{\mathfrak{c}_{\lambda}\right\}_{\lambda \in \Lambda}$ is uniquely defined.

Remarks 6.7. (a) The canonical basis defined above is the same as Lusztig's canonical basis. This is because the basis $\mathscr{E}$ is a PBW type basis (see [Ringel 1996, Theorem 7]). We also note that, as in the Hecke algebra case [Kazhdan and Lusztig 1979; 1980], the partial order used in this construction is the same as the one used in the geometric construction (see [Lusztig 1990, §9]).

(b) The relation (6-6) is derived via directed distinguished words. However, it can be used to prove the following result, ${ }^{2}$ which generalizes the formula given in Lemma 6.6 to all distinguished words. Thus we may also use nondirected distinguished words in the construction above to obtain canonical bases.

Proposition 6.8. For any distinguished word $w \in \Omega$, we have

$$
\delta(w)+\varepsilon(w)=-\operatorname{dim} M(\wp(w))+\operatorname{dim} \operatorname{End} M(\wp(w)) .
$$

Proof. Let $w \in \Omega$ be distinguished. By (6-2), we have

$$
\mathfrak{m}^{(w)}=v^{s} \tilde{\mathfrak{u}}_{\wp(w)}+\sum_{\lambda<\wp(w)} h_{\lambda, \wp(w)} \tilde{\mathfrak{u}}_{\lambda},
$$

where $s=\delta(w)+\varepsilon(w)+\operatorname{dim} M(\wp(w))-\operatorname{dim}$ End $M(\wp(w))$ and $0 \neq h_{\lambda, \wp(w)} \in \mathscr{L}$ for $\lambda<\wp(w)$. By applying $\iota$ to (6-7), we deduce from (6-6) that

$$
\iota\left(\mathfrak{m}^{(w)}\right)=v^{-s} \tilde{\mathfrak{u}}_{\wp(w)}+\sum_{\lambda<\wp(w)} d_{\lambda, \wp(w)} \tilde{\mathfrak{u}}_{\lambda}
$$

\footnotetext{
${ }^{2}$ We thank the referee for pointing out the proof.
} 
for some $d_{\lambda, \wp(w)} \in \mathscr{L}$. Since $\iota\left(\mathfrak{m}^{(w)}\right)=\mathfrak{m}^{(w)}$, equating coefficients yields $v^{s}=v^{-s}$. This implies $s=0$, that is,

$$
\delta(w)+\varepsilon(w)=-\operatorname{dim} M(\wp(w))+\operatorname{dim} \operatorname{End} M(\wp(w)) .
$$

\section{The type $A$ case}

We now give a combinatorial description of the map $\wp: \Omega \rightarrow \Lambda$ for the linear quiver

$$
Q=A_{n}: 1 \longrightarrow 2 \longrightarrow \cdots \longrightarrow n-1 \longrightarrow n \text {. }
$$

We also give an explicit description of the distinguished words in this case. Since $A_{n}$ is a subquiver of a cyclic quiver, the results obtained below and their proofs are similar to (or even simpler than) those given in [Deng and Du 2005], and the proofs will mostly be omitted.

It is known that, for $1 \leqslant i \leqslant j \leqslant n$, there is a unique (up to isomorphism) indecomposable $k A_{n}$-module $M_{i j}$ with top $S_{i}$ and of length $j-i+1$, and all $M_{i j}, 1 \leqslant i \leqslant j \leqslant n$, form a complete set of nonisomorphic indecomposable $k A_{n}$ modules. By Gabriel's theorem, each $M_{i j}$ corresponds to a positive root $\beta_{i j}$. Thus $\Phi^{+}=\left\{\beta_{i j} \mid 1 \leqslant i \leqslant j \leqslant n\right\}$. For each map $\lambda \in \Lambda$, we set $\lambda_{i j}=\lambda\left(\beta_{i j}\right)$. First, we have the following positivity result, which can be proved by counting and induction on the length of $w$ (compare [Deng and Du 2005, Proposition 9.1]).

Proposition 7.1. For each $w \in \Omega$ and each $\lambda \in \Lambda$, the polynomial $\varphi_{w}^{\lambda}$ lies in $\mathbb{N}[q]$.

Now, for each $i \in I$, we define a map $\sigma_{i}: \Lambda \rightarrow \Lambda$ as follows. For $\lambda \in \Lambda$, if $S_{i+1}$ is not a summand of $M(\lambda) / \operatorname{rad} M(\lambda)$ (i.e., $\lambda_{i+1, l}=0$ for all $l$ ), then $\sigma_{i} \lambda$ is obtained by adding 1 to $\lambda_{i i}$ so that $M\left(\sigma_{i} \lambda\right)=M(\lambda) \oplus S_{i}$; otherwise, $\sigma_{i} \lambda$ is defined by

$$
\left(\sigma_{i} \lambda\right)_{r s}= \begin{cases}\lambda_{r s} & \text { if }(r, s) \neq(i, j),(i+1, j), \\ \lambda_{i j}+1 & \text { if }(r, s)=(i, j), \\ \lambda_{i+1, j}-1 & \text { if }(r, s)=(i+1, j),\end{cases}
$$

where $j$ is the maximal index with $\lambda_{i+1, j} \neq 0$. We have the following (compare [Deng and Du 2005, Proposition 3.7]).

Proposition 7.2. Let $i \in I$ and $\lambda \in \Lambda$. Then $S_{i} * M(\lambda) \cong M\left(\sigma_{i} \lambda\right)$. Therefore $\wp(w)=\sigma_{i_{1}} \cdots \sigma_{i_{m}}(0)$ for any $w=i_{1} \cdots i_{m} \in \Omega$.

Let $w=j_{1}^{e_{1}} j_{2}^{e_{2}} \cdots j_{t}^{e_{t}} \in \Omega$ be in the tight form. For each $0 \leqslant r \leqslant t$, we put $w_{r}=j_{r+1}^{e_{r+1}} \cdots j_{t}^{e_{t}}$ and $\lambda^{(r)}=\wp\left(w_{r}\right)$. In particular, $w_{0}=w$ and $w_{t}=1$. Further, for $r \geqslant 1$, we have

$$
\lambda^{(r-1)}=\wp\left(w_{r-1}\right)=\underbrace{\sigma_{j_{r}} \cdots \sigma_{j_{r}}}_{e_{r}}\left(\lambda^{(r)}\right) .
$$


The following result gives a combinatorial description of distinguished words (compare [Deng and Du 2005, 5.5]).

Proposition 7.3. Let $w=j_{1}^{e_{1}} j_{2}^{e_{2}} \cdots j_{t}^{e_{t}} \in \Omega$ and $\lambda^{(r)}$, with $0 \leqslant r \leqslant t$, be given as above. Then $w$ is distinguished if and only if, for each $1 \leqslant r \leqslant t$, either $\lambda_{j_{r} j}^{(r)}=0$ for all $j_{r} \leqslant j \leqslant n$, or $e_{r} \leqslant \sum_{a=l_{r}+1}^{n} \lambda_{j_{r}+1, a}^{(r)}$ where $l_{r}$ is the maximal index for which $\lambda_{j_{r} l_{r}}^{(r)} \neq 0$.

Proof. Using a similar argument as in [Deng and Du 2005, Theorem 5.5], one can show that $w$ is distinguished if and only if, for each $1 \leqslant r \leqslant t, M\left(\lambda^{(r-1)}\right)$ admits a unique submodule isomorphic to $M\left(\lambda^{(r)}\right)$. However, the latter condition is equivalent to the described combinatorial condition, as shown in [Deng and Du 2005, Lemma 5.4].

\section{Acknowledgment}

The authors thank the universities of New South Wales and Virginia for their hospitality during the writing of the paper, and Brian Parshall for his comments on an early version of the paper.

\section{References}

[Bongartz 1996] K. Bongartz, "On degenerations and extensions of finite-dimensional modules", Adv. Math. 121:2 (1996), 245-287. MR 98e:16012 Zbl 0862.16007

[Chari and Xi 1999] V. Chari and N. Xi, "Monomial bases of quantized enveloping algebras", pp. 69-81 in Recent developments in quantum affine algebras and related topics (Raleigh, NC, 1998), edited by N. Jing and K. C. Misra, Contemp. Math. 248, Amer. Math. Soc., Providence, RI, 1999. MR 2001c:17023 Zbl 1054.17503

[Deng and Du 2005] B. Deng and J. Du, "Monomial bases for quantum affine $\mathfrak{s l}_{n}$ ”, Adv. Math. 191:2 (2005), 276-304. MR 2103214 Zbl 02128861

[Du 1994] J. Du, "IC bases and quantum linear groups", pp. 135-148 in Algebraic groups and their generalizations: quantum and infinite-dimensional methods (University Park, PA, 1991), edited by W. J. Haboush and B. J. Parshall, Proc. Sympos. Pure Math. 56, Amer. Math. Soc., Providence, RI, 1994. MR 95d:17010

[Gabriel 1972] P. Gabriel, “Unzerlegbare Darstellungen, I”, Manuscripta Math. 6 (1972), 71-103. Correction, ibid. 6 (1972), 309. MR 48 \#11212 Zbl 0232.08001

[Kashiwara 1991] M. Kashiwara, "On crystal bases of the $Q$-analogue of universal enveloping algebras”, Duke Math. J. 63:2 (1991), 465-516. MR 93b:17045 Zbl 0739.17005

[Kazhdan and Lusztig 1979] D. Kazhdan and G. Lusztig, "Representations of Coxeter groups and Hecke algebras”, Invent. Math. 53:2 (1979), 165-184. MR 81j:20066 Zbl 0499.20035

[Kazhdan and Lusztig 1980] D. Kazhdan and G. Lusztig, "Schubert varieties and Poincaré duality", pp. 185-203 in Geometry of the Laplace operator (Honolulu, 1979), edited by R. Osserman and A. Weinstein, Proc. Sympos. Pure Math. 36, Amer. Math. Soc., Providence, R.I., 1980. MR 84g:14054 Zbl 0461.14015 
[Lusztig 1990] G. Lusztig, "Canonical bases arising from quantized enveloping algebras", J. Amer. Math. Soc. 3:2 (1990), 447-498. MR 90m:17023 Zbl 0703.17008

[Reineke 2001a] M. Reineke, "Feigin's map and monomial bases for quantized enveloping algebras”, Math. Z. 237:3 (2001), 639-667. MR 2002f:17029 Zbl 1038.17008

[Reineke 2001b] M. Reineke, "Generic extensions and multiplicative bases of quantum groups at $q=0$ ”, Represent. Theory 5 (2001), 147-163. MR 2002c:17029 Zbl 1050.17015

[Ringel 1990] C. M. Ringel, "Hall algebras", pp. 433-447 in Topics in algebra, part 1: Rings and representations of algebras (Warsaw, 1988), edited by S. Balcerzyk et al., Banach Center Publ. 26, PWN, Warsaw, 1990. MR 93f:16027 Zbl 0778.16004

[Ringel 1993a] C. M. Ringel, "The composition algebra of a cyclic quiver: Towards an explicit description of the quantum group of type $\tilde{A}_{n}$ ", Proc. London Math. Soc. (3) 66:3 (1993), 507-537. MR 94g:16013 Zbl 0797.16014

[Ringel 1993b] C. M. Ringel, "Hall algebras revisited", pp. 171-176 in Quantum deformations of algebras and their representations (Ramat-Gan and Rehovot, 1991/1992), edited by A. Joseph and S. Shnider, Israel Math. Conf. Proc. 7, Bar-Ilan Univ., Ramat Gan, 1993. MR 94k:16021 Zbl 0852.17009

[Ringel 1995] C. M. Ringel, "The Hall algebra approach to quantum groups", pp. 85-114 in XI Escuela Latinoamericana de Matemáticas (Mexico City, 1993), edited by X. Gómez-Mont et al., Aportaciones Mat. Comun. 15, Soc. Mat. Mexicana, Mexico City, 1995. MR 96m:17034

[Ringel 1996] C. M. Ringel, "PBW-bases of quantum groups", J. Reine Angew. Math. 470 (1996), 51-88. MR 97d:17009 Zbl 0840.17010

Received August 15, 2003. Revised June 10, 2004.

\section{BANGMING DENG \\ DePartment of Mathematics \\ BEIJING NORMAL UNIVERSITY \\ BEIJING 100875 \\ CHINA}

dengbm@bnu.edu.cn

JIE DU

SCHOOL OF MATHEMATICS

UNIVERSITY OF NEW SOUTH WALES

UNSW SYDNEY NSW 2052

AUSTRALIA

j.du@unsw.edu.au

http://www.maths.unsw.edu.au/ jied 